\title{
Plattformbasierte Dienstleistungen
}

\section{Dienstleistungen als Treiber des gesellschaftlichen Wandels}

\author{
Katinka Weissenfeld, Angelina Dungga und Jan Frecè
}

\begin{abstract}
Zusammenfassung
Peer-to-Peer-Dienstleistungsplattformen (P2P-Plattformen) stellen eine besondere Form von Dienstleistungsplattformen dar, die z. B. Geschäftsmodelle der Sharing Economy in seiner heutigen Form ermöglichen. Als Reaktion auf den aktuell herrschenden Begriffsdschungel rund um Sharing Economy und Dienstleistungsplattformen im Allgemeinen werden zunächst Begrifflichkeiten geklärt, bevor Aspekte des gesellschaftlichen Wandels diskutiert werden. Aufgrund iterativer Wechselwirkungen zwischen Sozialstrukturen und sozialen Akteuren werden die technischen, sozialen, ökologischen und ökonomischen Aspekte des gesellschaftlichen Wandels sowohl als Folge als auch als Vorbedingungen für das Aufkommen von P2P-Plattformen betrachtet. Abschließend werden in einem dritten Teil Chancen und Herausforderungen diskutiert, die sich aktuell und zukünftig durch P2P-Plattformen für die Gesellschaft ergeben. Dabei ist festzustellen, dass viele Aspekte sowohl Chancen als auch Herausforderungen mit sich bringen, die entsprechend abzuwägen sind.
\end{abstract}

K. Weissenfeld $(\varangle) \cdot$ A. Dungga $\cdot$ J. Frecè

Berner Fachhochschule Wirtschaft, Bern, Schweiz

E-Mail: katinka.weissenfeld@bfh.ch

A. Dungga

E-Mail: angelina.dungga@bfh.ch

J. Frèce

E-Mail: jan.frece@bfh.ch

J. Schellinger et al. (Hrsg.), Digitale Transformation und Unternehmensführung,

https://doi.org/10.1007/978-3-658-26960-9_3 


\subsection{Einleitung}

Das Phänomen eines gesellschaftlichen Wandels durch die Digitalisierung lässt sich durch das Zitat von Stalder (Stalder 2016) sehr gut wiedergeben: „Erst heute, wo die Faszination für die Technologie abgeflaut ist und ihre Versprechungen hohl klingen, wird die Kultur und Gesellschaft in einem umfassenden Sinne durch Digitalität geprägt“".

Der vorliegende Beitrag befasst sich mit plattformbasierten Dienstleistungen als Treiber für gesellschaftlichen Wandel und zeigt auf, welche Chancen und Herausforderungen für die Gesellschaft damit verbunden sind. Aufgrund der Vielfältigkeit und Komplexität plattformbasierter Dienstleistungen widmet sich der Beitrag digitalen Plattformen, die den direkten Bezug oder Austausch von Waren und Dienstleistungen unter „peers “, also unter gleichgestellten Mitgliedern einer Gruppe ermöglichen. Diese sind Ausdruck einer speziellen Form der Wirtschaft, die gesellschaftliche Veränderungen impliziert und von der ein beträchtliches Wachstum ausgeht. So wird in der EU der Bruttoumsatz solcher Plattformen im Jahr 2015 auf 28 Mrd. EUR geschätzt und es wird eine Verdoppelung des Umsatzes im Vergleich zum Vorjahr in fünf Schlüsselbranchen ${ }^{1}$ festgestellt. Des Weiteren geht die EU von einem zukünftigen Wachstumspotenzial entsprechender Plattformen von 160 bis 572 Mrd. EUR aus (2016). Dies zeigt die große Bedeutung und hohe Relevanz solcher Plattformen für die Gegenwart, aber auch für die nahe Zukunft.

In einem ersten Teil des vorliegenden Beitrags wird zunächst auf die Begriffsklärung von Peer-to-Peer-Dienstleistungsplattformen (P2P-Dienstleistungsplattformen) eingegangen, um so ein einheitliches Verständnis für die nachfolgenden Inhalte zu ermöglichen. Anschließend werden in einem zweiten Teil wesentliche Aspekte beschrieben, die in Interaktion mit P2P-Dienstleistungsplattformen von gesellschaftlicher Relevanz sind. In einem abschließenden dritten Teil stehen Chancen und Herausforderungen im Fokus der Diskussion, denen eine entsprechend hohe gesellschaftliche Brisanz zugesprochen werden und die für eine bestmögliche Entwicklung von ökologischen, gesellschaftlichen und wirtschaftlichen Potenzial von P2P-Dienstleistungsplattformen von Relevanz sind.

\subsection{Peer-to-Peer-Dienstleistungsplattformen}

Dienstleistungen haben über die letzten 60 Jahre hinweg einen zentralen Platz in unserer Gesellschaft eingenommen. Wie bereits in den 1950er-Jahren in der Drei-Sektor-Theorie von Fourastié (1954) beschrieben, gewinnt der tertiäre Sektor, nämlich die Dienstleistung, immer mehr an Bedeutung und dominiert unsere Arbeitswelt vor den primären und sekundären Sektoren Rohstoffgewinnung und Rohstoffverarbeitung. Mittlerweile

\footnotetext{
${ }^{1}$ Als Schlüsselbranchen gelten: Unterkunft (Kurzzeitvermietung), Personenbeförderung, Dienstleistungen für private Haushalte, freiberufliche und technische Dienstleistungen sowie Schwarmfinanzierung (Crowdfunding).
} 
spricht man von einem vierten Sektor (Gottmann 1957) dem Informationssektor, da die Dienstleistungen einen solchen Umfang eingenommen haben, dass gewisse Disziplinen den Bedarf sehen, diese entsprechend aufzuteilen. Der quartäre Sektor ist terminologisch nicht präzise fassbar, deckt aber gemäß Redlich und Wulfsberger (2011) immaterielle Güter wie Informationen, Ideen und Daten ab. Die Kommunikation, Speicherung und Verarbeitung dieser Daten und Informationen ist stark durch die heutigen technologischen Möglichkeiten geprägt (Traiblmaier 2006).

Im Zuge der Digitalisierung können Dienstleistungen, d. h. sowohl personenbezogene Dienstleistungen, wie z. B. Beratungsdienste, Taxi- und Hoteldienste, Weiterbildung und Verwaltungsdienste als auch sachbezogene Dienstleistungen wie Überbrückungsdienste in Form von Transport und Erhaltungsdienste in Form von Wartung und Reparaturen (Bodendorf 1999), immer einfacher einer Vielzahl von Nutzern angeboten werden. Ein weiterer stark zu beobachtender Trend besteht darin, dass Dienstleistungen nicht nur von professionellen Betreibern sondern von Privatpersonen über das Internet angeboten werden. Mittlerweile existieren zahlreiche Plattformen im Internet, über die Privatpersonen mit Internetzugriff kostengünstig und ohne größere Aufwände und Risiken ihre Dienstleistungen unzähligen potenziellen Kunden weltweit anbieten können. Die notwendigen Transaktionen zwischen Leistungserbringer und -bezieher können dabei ohne Einbezug eines Dritten durchgeführt werden.

Wird eine Internetplattform genutzt, um Dienstleistungen $\mathrm{zu}$ präsentieren und einen Leistungserbringer und einen Leistungsbezieher $\mathrm{zu}$ vermitteln, spricht man von einer Dienstleistungsplattform. Hier wird zunächst nicht unterschieden, ob es sich bei Leistungserbringer und -bezieher um Unternehmen, staatliche Einrichtungen oder Privatpersonen handelt. Des Weiteren kann die Rolle des Plattformbetreibers eine reine Vermittlerrolle oder aber auch eine unterstützende Rolle bei der Leistungserbringung sein (z. B. Unterstützung bei der Bezahlung). Beispiele für Dienstleistungsplattformen sind Online-Schalter von Behörden oder Immobilienplattformen wie immoscout24, aber auch Facebook als Kommunikationsplattform und Uber oder Airbnb können unter dem Begriff der Dienstleistungsplattform zusammengefasst werden.

Da die digitale Transformation und die damit einhergehende Vielfalt an Dienstleistungsplattformen neue Möglichkeiten für den geschäftlichen Austausch zwischen Gleichgestellten (Peers), d. h. i. d. R. Privatpersonen, ermöglicht, stützen sich die Ausführungen in Tab. 3.1 auf den vorherrschenden Trend $\mathrm{zu}$ sogenannten P2P-Dienstleistungsplattformen.

Von P2P-Dienstleistungsplattformen spricht man, wenn es sich um dezentrale Plattformen handelt, bei denen Leistungserbringer und Leistungsbezieher zumindest bei der Leistungserbringung direkt und ohne Einbinden eines Dritten agieren. Während traditionelle Geschäfte stark durch Economies of Scale und die Massenproduktion getrieben werden, stehen beim P2P-Business aus Sicht der Beteiligten zunehmend die Economies of Scope im Vordergrund (Quinn 1992). Die Effizienz entsteht hierbei, indem bei der Leistungserbringung zwischen Leistungserbringer und Leistungsbezieher keine Dritten im primären Prozess involviert werden. Bei einem P2P-Business spricht man im 
Tab. 3.1 Begriffsdefinitionen zu Peer-to-Peer. (Eigene Darstellung)

\begin{tabular}{l|l}
\hline Begriff & Eigenschaften und Beispiele \\
\hline Peer-to-Peer (Peers) & $\begin{array}{l}\text { Die Akteure (Peers) weisen eine hohe Heterogenität auf (beide } \\
\text { Akteure sind meistens Privatpersonen) } \\
\text { Peers bieten Dienste an und beziehen auch Dienste } \\
\text { Die Peers agieren autonom und sind selbstorganisiert }\end{array}$ \\
\hline Peer-to-Peer-Business & $\begin{array}{l}\text { Bei der Leistungserbringung zwischen Leistungserbringer und } \\
\text { Leistungsbezieher ist kein Dritter involviert } \\
\text { Leistungserbringer und Leistungsbezieher von Gütern oder } \\
\text { Dienstleistungen agieren unabhängig von anderen Parteien mit- } \\
\text { einander }\end{array}$ \\
\hline Peer-to-Peer-Dienstleistungen & $\begin{array}{l}\text { Dienstleistungen, die direkt zwischen Leistungserbringer } \\
\text { und Leistungsbezieher - ohne Einbezug eines Dritten - aus- } \\
\text { getauscht werden } \\
\text { Beispiele: Beratungsdienste, Fahrdienste, Übernachtungs- } \\
\text { dienste, Weiterbildungsdienste, Finanzdienste, Überbrückungs- } \\
\text { dienste wie Transport und Erhaltungsdienste wie Wartung und } \\
\text { Reparaturen }\end{array}$ \\
\hline Peer-to-Peer-Plattform & $\begin{array}{l}\text { Technologie, die P2P-Dienstleistungen unabhängig von Ort } \\
\text { und Zeit unterstützt, d. h. die Kommunikation zwischen den } \\
\text { Akteuren (Peers) und die Präsentation der Dienstleistung im } \\
\text { Internet ermöglicht } \\
\text { Beispiele: ebay, Airbnb }\end{array}$ \\
\hline
\end{tabular}

Gegensatz zum traditionellen Geschäft daher auch von einem dezentralen Geschäft, bei dem Anbieter und Bezieher von Gütern oder Dienstleistungen unabhängig von anderen Parteien direkt miteinander agieren (aber allenfalls über entsprechende elektronische Plattformen von Dritten entsprechend vermittelt).

Prominente Beispiele hierfür sind eBay und Airbnb, bei denen es sich um Plattformen handelt, die Dienstleistungen (oder Waren) ohne das Einbinden eines Dritten in der Phase der Leistungserbringung ermöglichen.

Diese Art des Geschäfts erinnert stark an das vorindustrielle Zeitalter, in dem Einzelhändler ihre eigens produzierte Ware direkt an Kunden verkauften (etwa durch die Eliminierung teurerer persönlicher Makler). Die digitale Transformation ermöglicht nun, dieses eigentlich veraltete Businessmodell in einigen Bereichen auf eine neue Weise wiederzubeleben. Wichtige und neue Akteure für das Business-Modell von P2P-Dienstleistungen sind die Plattformbetreiber, die nicht direkt in den Leistungserbringungsprozess involviert sind, jedoch essenzielle Grundlage für P2P-Dienstleistungen schaffen. Mit den heute existierenden P2P-Plattformen wurde eine effiziente und moderne Lösung geschaffen, die P2P-Dienstleistungen unabhängig von Ort und Zeit ermöglicht (Chtacherbia et al. 2002). 
Bei den heutigen P2P-Plattformen unterscheidet man die folgenden Ausprägungen (Botsman und Rogers 2010):

- die Akteure Leistungserbringer und Leistungsbezieher handeln ökonomisch getrieben und nutzen die P2P-Plattform, um kommerzielle Geschäfte abzuwickeln, wie dies beispielsweise bei eBay der Fall ist;

- Plattformen, bei denen Leistungserbringer und Leistungsbezieher nicht nur rein ökonomisch getrieben handeln, sondern auch weltanschauliche Aspekte wie etwa das Teilen und damit das Schonen von Ressourcen ebenfalls im Vordergrund stehen. Diese Form von P2P-Plattformen wird häufig auch als Sharing-Economy-Plattform bezeichnet (Botsman und Rogers 2010). Beispiele hierzu sind u. a. Airbnb und BlaBlaCar. Zur Sharing-Economy zählen neben dem Aspekt des Teilens auch das Tauschen und Leihen von Dienstleistungen und Produkten (Keller 2013);

- unterschiedliche Personen werden über eine P2P-Plattform vernetzt, um gemeinsam an Projekten zu arbeiten (z. B. OpenInnov);

- es wird Wissen über eine Plattform zur Verfügung gestellt, das von jedem abgerufen werden kann und damit geteilt wird (z. B. Wikipedia);

- Kommunikationsplattformen, wie beispielsweise Facebook, Retroshare oder Tox.

Im Folgenden wird auf alle genannten Ausprägungen Bezug genommen. Im Fokus stehen jedoch Plattformen, über die kommerzielle Geschäfte abgewickelt werden und bei denen eine neue Art der Geschäftsbeziehung durch Teilen entsteht.

\subsection{Aspekte gesellschaftlichen Wandels in Interaktion mit Peer-to-Peer-Dienstleistungsplattformen}

Das Aufkommen von P2P-Dienstleistungsplattformen beeinflusst eine Gesellschaft in verschiedener Hinsicht und führt dabei einen Wandel in Verhalten und Wahrnehmung vieler Menschen herbei. Dieser Wandel geht in einer modernen Gesellschaft so weit, dass von einer Veränderung der Struktur eines sozialen Systems die Rede sein kann, also von einem gesellschaftlichen Wandel. ${ }^{2}$

Nach einer sozialen Innovation durch P2P-Dienstleistungen, tritt der gesellschaftliche Wandel schrittweise durch ein Wechselspiel von bestehender Struktur des sozialen Systems und neu auftretendem Verhalten ein. P2P-Dienstleistungsplattformen sind also nicht nur Ausdruck gesellschaftlichen Wandels, sondern gleichzeitig dessen Vorbedingung.

\footnotetext{
${ }^{2}$ Aus den zahlreichen Definitionen des Begriffs „Gesellschaftlicher Wandel“ wird hier derjenige von Giddens (Hamari et al. 2016) und Stones (Weber 1922) verwendet, da dieser die Wechselwirkung zwischen Agenten und Struktur besonders herausarbeitet.
} 
Da Mitglieder sozialer Gemeinschaften häufig ähnliche Lebenswelten pflegen und sich diese gegenseitig bestätigen und somit auch ein „Wir“-Gefühl schaffen (Hillmann 1994), findet Fortschritt innerhalb einer Gesellschaft i. d. R. gruppenweise statt. Die nachfolgenden Ausführungen adressieren folglich nie die Gesamtheit einer Gesellschaft, sondern immer nur gesellschaftliche Teilgruppen und ihre Perspektive(n) auf einmal.

Wie gesellschaftlicher Wandel sich im Einzelnen entwickeln wird, ist im Voraus kaum abzuschätzen, aber es ist mit einiger Sicherheit vorauszusagen, welche Aspekte in der Ausgestaltung des gesellschaftlichen Wandels von Relevanz sein werden. Aus diesem Grund beschreiben die nachfolgenden Abschnitte eine Vielzahl von Aspekten, die im Zusammenhang mit P2P-Dienstleistungsplattformen einen gesellschaftlichen Wandel vorantreiben können. Zunächst werden technologisch ausgerichtete Themen wie Nutzerzahlen und Vertrauen in die P2P-Dienstleistungsplattformen thematisiert. AnschlieBend werden die sozialen Voraussetzungen und die beispielhaften Mechanismen des gesellschaftlichen Wandels beschrieben bevor auf die potenziellen ökologischen Folgen und die ökonomischen Aspekte, d. h. die Auswirkungen der neuen Dienstleistungen auf das Marktangebot, eingegangen wird.

\subsubsection{Technologische Aspekte}

Die Möglichkeit für plattformbasierte Dienstleistungen ist primär durch den technologischen Wandel der letzten Dekaden gegeben. Zum einen ist neben den technischen Voraussetzungen die nötige internationale Akzeptanz und Verbreitung der Technologien vorhanden. Zum anderen gewinnt mit dem Aspekt der technologischen Entwicklung die Sicherstellung von Qualität und Vertrauen eine neue Dimension. Im Folgenden werden die steigenden Nutzerzahlen von digitalen Plattformen im Detail beleuchtet und der Aspekt von Qualität und Vertrauen bezüglich digitaler Plattformen diskutiert.

\subsubsection{Steigende Nutzerzahlen von digitalen Plattformen}

Die International Telecommunication Union (ITU) publiziert jährlich Zahlen zur IKT-Nutzung weltweit. Die neuesten Daten zeigen, dass im Jahre $2017 \mathrm{mehr}$ als zwei Drittel der Weltbevölkerung in Gebieten leben, die über eine hohe mobile Breitbandabdeckung verfügen. Zudem werden IKT-Leistungen immer preiswerter. Ca. $80 \%$ der Bevölkerung in Industriestaaten nutzen das Internet, wobei der Anteil der Haushalte mit Internetanschluss sogar bei über $80 \%$ liegt. Wird die Anzahl Registrierungen an das mobile Netz betrachtet, so sind ungefähr $90 \%$ der Bevölkerung aus Industriestaaten an mobile Netze angeschlossen (ITU 2017).

Zahlen der OECD bestätigen zudem, dass in den OECD-Ländern eine Verlagerung der Wirtschaft in das Internet stattfindet (OECD 2014). Ca. $65 \%$ der 25- bis 44-jährigen Internetnutzer haben bereits Waren oder Dienstleistungen über das Internet bestellt, wobei die Top-3-Länder (Großbritannien, Deutschland, Dänemark) einen Anteil von ca. $90 \%$ ausmachen. Auch bei den über 65-Jährigen führten im Jahr 2013 ca. $40 \%$ 
eine Bestellung über das Internet durch. Wird der Anteil der E-Commerce-Teilnehmer an der gesamten Bevölkerung gemessen, so kann zwischen 2007 und 2013 ein Wachstum von ca. 18 Prozentpunkten festgestellt werden. Werden die Unternehmen nach Unternehmensgröße heruntergebrochen, wird ersichtlich, dass v. a. große Unternehmen (>250 Mitarbeiter) Online-Transaktionen anbieten. Gleichzeitig hält der Trend zur höheren Nutzung von Smartphones an und überholte 2013 die Nutzung von herkömmlichen stationären Telefonen. Dieser Trend scheint sich stark auf Dienstleistungsplattformen auszuwirken. So stieg die Nutzung von Facebook via Smartphone von $28 \%$ aller Facebook-Nutzer auf $75 \%$ im Jahre 2013. Dieser Trend wird auch durch die Nutzungszahlen von Wikipedia bestätigt. Der Zugriff via Smartphone auf die Webseite lag im Juni 2010 bei fast Null und stieg auf über 4 Mrd. Ende des Jahres 2013. Der Anteil an Zugriffen über Smartphones steigt im Vergleich zu anderen Kanälen heute stetig (OECD 2014).

Die weite Verbreitung des Internets und die Verlagerung wirtschaftlicher Kommunikationen und Transaktionen in das Internet legen nahe, dass auch P2P-Dienstleistungsplattformen von dieser Entwicklung profitieren. Ebenfalls ist zu berücksichtigen, dass Dienstleistungsplattformen zu jeder Zeit und von jedem Ort erreichbar seien können, da viele Nutzer die Möglichkeiten über das Smartphone verstärkt nutzen.

\subsubsection{Qualität und Vertrauen auf digitalen Plattformen}

Vertrauen ist ein multidimensionaler und subjektiver Faktor, der sowohl für Leistungsempfänger als auch für Leistungserbringer eine zentrale Rolle spielt, wenn Dienstleistungen (online) bezogen werden. Im Zuge der Digitalisierung werden Dienstleistungen immer häufiger über das Internet angeboten, sodass Leistungsempfänger und Leistungserbringer sich nicht persönlich kennenlernen und teilweise ausschließlich virtuelle Geschäfte abwickeln. In der Regel existieren Verträge und mögliche Reputationen über die Leistungsempfänger und Leistungserbringer, die das Vertrauen in den jeweiligen Geschäftspartner und damit in die Qualität der Dienstleistung der Leistungserbringer respektive in die Zahlungsmoral der Leistungsempfänger stärken sollen.

Werden Dienstleistungen über P2P-Plattformen angeboten, können die Geschäftspartner gegenseitiges Vertrauen nur virtuell aufbauen. Hierzu existieren bereits viele verschiedene Hilfsmittel, so etwa das Bewerten von Leistungsempfänger und Leistungserbringer und deren Leistungen oder aber allenfalls Gütesiegel, die über eine Zertifizierung durch Externe vergeben werden. Die Bewertungen durch Leistungsempfänger und Leistungserbringer finden für jede Plattform separat statt und sind nur auf der jeweiligen Plattform an die virtuelle Identität des einzelnen Leistungsempfängers und Leistungserbringers geknüpft. Über die Qualität der Dienstleistung oder der Ware ist ebenfalls nur das bekannt, was der Leistungserbringer in seiner subjektiven Wahrnehmung darüber berichtet.

So werden Angebote der sogenannten Sharing Economy, die auf dem kollaborativen Lebensstil basiert, beispielsweise nur von rund $20 \%$ der Bevölkerung genutzt, obwohl das Potenzial viel höher ist. Hauptbedenken der Nutzer ist die Unsicherheit und das damit nicht vorhandene Vertrauen in den jeweiligen Leistungserbringer bzw. 
Leistungsempfänger (BITKOM 2013). Des Weiteren wird der Bedarf nach Regulierungen und Standards für den neuen Markt der Sharing Economy immer größer, d. h. dass auch Standards für Reputationen und damit für die Vertrauenswürdigkeit der Akteure ein wichtiger Erfolgsfaktor für ein gesichertes Wachstum der Sharing Economy bzw. P2P-Dienstleistungsplattformen ist. $\mathrm{Zu}$ bedenken ist hierbei jedoch die enorme Vielfalt an P2P-Dienstleistungsplattformen, für die eine einheitliche Regulierung und Standardisierung über eine Vielzahl von Rechtsräumen hinweg kaum möglich ist. Eine Regulierung, die Gültigkeit für bestimmte Sektoren hat und für Online- und physische Dienstleistungen gleich ist, scheint eine angemessene Vision für die Zukunft zu sein (level playing field) (BMWi Bundesministerium für Wirtschaft und Energie 2016).

\subsubsection{Soziale Aspekte}

Grob definiert besteht gesellschaftlicher Wandel aus einer Iteration zweier Elemente:

1. Soziale Akteure reagieren auf die kulturelle, politische oder soziale und natürliche Umwelt betreffend Wandel mit neuen Weltanschauungen, Rollenvorstellungen etc.

2. Bestehende soziale Institutionen und konservative soziale Akteure reagieren auf neuartige Ideen und Handlungen.

Die wiederholte Wechselwirkung dieser beiden Elemente führt dabei zu einem gesellschaftlichen Prozess und im Idealfall schlussendlich zu einem gesellschaftlich stabilen Kompromiss. Wie stark in diesem spezifischen Fall

- die Interessen der Nutzer (billigere und/oder bessere Dienstleistung),

- die Interessen des jeweiligen Gewerbes (Marktanteil halten, bisheriges Geschäftsmodell ausschöpfen) oder

- die Interessen des Staates (klare legale Regelung, verlässliche Steuereinnahmen in genügender Höhe, soziale Ruhe) gewichtet werden, ist eine Frage der Machtdisparität verschiedener Gruppen einer Gesellschaft und ihrer Fähigkeit und Bereitschaft, Kompromisse einzugehen.

Die nachfolgenden Kapitel beleuchten für das Beispiel von P2P-Dienstleistungsplattformen relevante Elemente gesellschaftlichen Wandels: Das erste Kapitel schildert die gesellschaftlichen Vorbedingungen, welche dem Aufkommen von Communities im 20. und 21. Jahrhundert überhaupt erst den Boden bereitet haben, während sich die beiden folgendem Kapitel dem Einfluss von und der Teilhabe an technologischem Fortschritt zuwenden. Das letzte Kapitel beschreibt schließlich institutionellen Wandel am Beispiel von P2P-Dienstleistungsplattformen und zeigt beispielhaft davon betroffene Institutionen auf. 


\subsubsection{Stärkung des Community-Gedankens durch Stärkung des Einzelnen}

Die Gemeinschaft ist die ursprünglichste Zusammenlebensform des Menschen und damit das historische Grundelement jeder Gesellschaft. Der Begriff der Gemeinschaft, obwohl zuerst geprägt von Tönnies (1887), wird heute in diesem Zusammenhang eher im Sinn von Weber verwendet und bezeichnet somit eine soziale Beziehung, ,wenn und soweit die Einstellung des sozialen Handelns ... auf subjektiv gefühlter (affektueller oder traditionaler) Zusammengehörigkeit der Beteiligten beruht“ (Weber 1922). Im Gegensatz dazu stellt der Begriff der Gesellschaft eine soziale Beziehung dar, ,wenn und soweit die Einstellung des sozialen Handelns auf rational (wert- oder zweckrational) motiviertem Interessenausgleich oder Interessenverbindung beruht“ (Weber 1922). Beide Phänomene kommen allerdings nur in seltensten Fällen in ihrem „ganz reinen Typus“ (Weber 1922) vor, sodass Gemeinschaften z. B. zweckrationale Motivationen aufweisen dürfen, ohne dadurch als eine Vergesellschaftung zu gelten.

Das heute zu beobachtende Erstarken der Gemeinschaft in Form von Online Communities (Kardorff 2006) ist maßgebend durch folgende soziale Phänomene ermöglicht und/ oder vorangetrieben:

- Ein Großteil der Bevölkerung ist 24/7 mit dem Internet verbunden (Eurostat 2015). Dies ermöglicht eine breite und alltägliche Nutzung der Innovation.

- Eigentum als traditionelles Statussymbol verliert beständig an Bedeutung (Belk 2014). Erst durch den beginnenden sozialen Bedeutungsverlust von Eigentum zugunsten von funktionalem Besitz ${ }^{3}$ und vor allem Zugang, kann Teilen sozial akzeptabel werden.

- Individualisierte Lebensführung wird höher gewertet als sozial akzeptierte Lebensentwürfe und Lebensläufe (Beck 1986; Dahrendorf 1961; Thome 1985). Traditionelle Anbieter haben dadurch nicht per se den Vorzug vor neuartigen Angeboten.

- Von Dienstleistungsplattformen akzeptierte Zahlungsmittel sind großflächig im Einsatz. Eine Teilnahme an diesen Plattformen ist dadurch praktisch ohne Zusatzaufwand (Registration auf der Plattform) möglich.

- Ressourcenverbrauch wird nicht mehr im selben Maß mit Status verbunden (Weller 2015). Recycling und effizientere Nutzung von Ressourcen wird - zunächst noch als fortschrittliches Verhalten gewertet - zunehmend zum Mainstream, was den Erfolg von P2P-Dienstleistungsplattformen befeuert. Würde Verbrauch immer noch als Ausdruck von Erfolg und Reichtum gesehen, wäre diesen Plattformen ein entsprechendes soziales Stigma sicher.

Die Tatsache, dass eine verstärkte Individualisierung erst ein Erstarken von Communities ermöglicht, erscheint auf den ersten Blick paradox, sollte doch das „Wir“ bei Communities

\footnotetext{
${ }^{3}$ Unter funktionalem Besitz wird hier der Wille verstanden, über einen Gegenstand zu verfügen, um ihn zu gebrauchen.
} 
im Fokus stehen und nicht das „Ich“. Dabei ist zu bedenken, dass die Existenz einer Vielzahl unterschiedlicher, zum Teil selbstverwalteter Communities, die nach Belieben entstehen und vergehen können, einen fundamentalen Bruch mit den sozialen Konventionen in der Vorstellung der 1950er- und 1960er-Jahre des 20. Jahrhunderts und den allermeisten vorhergehenden Sozialkonventionen darstellt. Dieser Bruch wurde dadurch ermöglicht, dass das Individuum in der Betrachtung der Gesellschaft an Wichtigkeit gewann und vermehrt als einzelne Persönlichkeit und nicht als bloße Manifestation der Gemeinschaft oder später Gesellschaft gesehen wurde.

Erst als die individuelle Persönlichkeit unabhängig genug sein durfte, um subjektiv ein effektuiertes oder traditionelles Zusammengehörigkeitsgefühl zu entwickeln - zuvor wurden solche Tendenzen als gefährliche soziale Anomalien gesehen, die es im Auge zu behalten galt -, wurde die Bildung von Communities und eventuell darin gelebter Subkulturen sozial denkbar und machbar. In Kombination mit neuen Technologien, welche die Bildung einer Online Community unabhängig von einem geografischen Ort ermöglichen, aber auch einer voranschreitenden Technisierung früher technikfernerer Gruppen, rückten globale Communities aus dem Bereich des Denkbaren ins Machbare.

\subsubsection{Technischer Fortschritt und gesellschaftlicher Wandel}

Neues Wissen oder technische Errungenschaften ziehen seit Jahrhunderten einen Wandel der davon betroffenen Gemeinschaften und Gesellschaften nach sich. Berufszweige entstehen und verschwinden, soziale Wertzuschreibungen verschieben sich, selbst der Stellenwert von religiösen Weltanschauungen kann von Fortschritten in Technik und Wissen beeinflusst werden.

Kurzfristige, tief greifende technische Innovationen, wie sie P2P-Dienstleistungsplattformen zweifellos darstellen, durchbrechen nicht nur Rollenerwartungen, sondern können völlig neue Ansprüche an Lernende stellen, die von einigen Mitgliedern der Gruppe potenziell nicht mehr oder nur unter zusätzlichem Aufwand zu leisten sind. Vor allem grundlegend innovative Entwicklungen, welche nicht nur eine leichte Anpassung des Bekannten, sondern tief greifend neue, vielleicht auch kontraintuitive Perspektiven und Denkweisen voraussetzen, verlangen Adaptionsfähigkeit. Im Versagensfall wird das Nichtadaptieren entsprechender Denkweisen mit Statusverlust oder gar sozialem Ausschluss sanktioniert.

Eine qualitative Änderung des gesellschaftlichen Wissenskorpus bedeutet auf der einen Seite immer den Statusverlust der bis dahin Wissenden und stellt für die Stabilität der gesellschaftlichen Struktur eine Gefährdung dar. Auf der anderen Seite bietet das neue Wissen denjenigen, die das neue Wissen aufzunehmen und zu nutzen wissen, neue Chancen sozial oder ökonomisch aufzusteigen (Mead 1934). Sozial machtvollen Institutionen oder Individuen kann folglich nur an gesellschaftlicher Veränderung liegen, wenn sie sich zutrauen, die Veränderung zu ihrem Vorteil zu nutzen. Je nach Macht dieser Institutionen oder Personen kann es in der Folge zu Einschränkungen der Nutzung oder Verbreitung neuen Wissens und/oder einer Subvention herkömmlichen Wissens kommen. 
Gilt diese Einschränkung nicht vollständig, sickert die Innovation über die Early Adopter einer Gesellschaft langsam durch. Wenn auch der Gewinn an sozialem Status häufig nicht sofort einsetzt, da die ,guten alten Dinge“ noch eine Weile mit einem Nostalgievorteil im sozialen Rennen verbleiben, setzen die funktionalen Vorteile der Nutzung einer neuen Technologie unmittelbar ein. Menschen, welche das neue Wissen adaptiert und die Anpassung gemeistert haben, erhalten durch die Anwendung des neuen Wissens Vorteile. Diese werden von anderen erkannt (Mead 1934). Je bekannter und beliebter ein Anwender neuer Technologie ist und je zugänglicher und übertragbarer die durch die neue Technologie hervorgebrachten Vorteile sind, desto mehr Menschen schreiben der neuen Technologie positive Eigenschaften zu und empfinden sie als erstrebenswert (Hill 2010). Dies geschieht auch gegen den geltenden sozialen Konsens, was diesen wiederum schrittweise schwächt. Ist gesellschaftlicher Wandel einmal so weit vorgedrungen, ist es nicht mehr Macht, die Wissen beschränkt, sondern neues Wissen, das bestehende Machtverhältnisse ins Wanken zu bringen vermag. Es ist also sehr wahrscheinlich, dass tief gehenden Innovationen Widerstand von jenem Teil der Gesellschaft erhalten, der durch einen Wandel der Verhältnisse an Macht und Status verliert.

$\mathrm{Ob}$ eine Gesellschaft den potenziellen Ausschluss einzelner Gruppen als Preis für die Inklusion oder den Aufstieg anderer Gruppen in Kauf nimmt, hängt ab von relativen Faktoren, wie z. B. der Größe, Reputation und dem Machtpotenzial der betroffenen Gruppen, aber auch absoluten Aspekten, wie z. B. dem Selbstverständnis der urteilenden Bevölkerungsgruppen, legalen Standards, zur Verfügung stehenden Ressourcen, sozialen Traditionen usw. (Warschauer 2004; The Tomás Rivera Policy Institute 2015). Jede Gesellschaft steht in all ihrer Einzigartigkeit der gleichen Herausforderung des gesellschaftlichen Wandels gegenüber: Der Modernisierung von Institutionen, ohne dass aus Gründen des Machterhalts Teile von der Modernisierung ausgeschlossen werden und ohne dass Teile der Gesellschaft aufgrund ihrer traditionellen Haltung sanktioniert werden. Breite soziale Teilhabe ist für offene Gesellschaften zentral, um ein Wir-Gefühl zu schaffen und zu erhalten, das den Zusammenhalt offener Gesellschaften langfristig überhaupt erst ermöglicht (Popper 1975).

Einerseits ermöglichen P2P-Dienstleistungsplattformen eine Privatisierung der Dienstleistungserbringung, was insbesondere für eine Dienstleistungsgesellschaft eine Art teilweise Vergemeinschaftung der Gesellschaft bedeutet. Damit ist zwar nicht eine De-facto-Rückkehr zur gemeinschaftlichen Lebensform gemeint, sondern das Phänomen, dass der Handelspartner trotz des zumindest anfänglich klar funktionalen Beziehungscharakters sein Gegenüber nicht primär als Rollenvertreter einer Institution ansieht, dem an der Bewahrung der bestehenden Verhältnisse liegt, sondern als Privatperson wie sich selbst, daher der Begriff „Peer“ (Deaux 1996).

\subsubsection{Institutionen im gesellschaftlichen Wandel}

Die Identifikation und Trennung von privaten und juristischen Personen ist in den sozialen Institutionen Europas seit Jahrhunderten institutionell verankert, um der jeweiligen 
Rolle und den damit verbundenen sozialen, aber auch rechtlichen Erwartungen gerecht werden zu können. Die Aufteilung in private und gewerbliche Handlungen und deren Regelung hat historischen Hintergrund, ist somit keine soziale Konstante und daher durch gesellschaftlichen Wandel jederzeit umformbar. So existiert bei P2P-Dienstleistungsplattformen solch eine klare Unterteilung zwischen privatem und gewerblichem Handeln nicht mehr. Beispielsweise ist das Vermieten der eigenen (Ferien-)Wohnung an Freunde und Bekannte, das Verleihen des eigenen Autos oder das Teilen von Fahrtkosten durch Fahrgemeinschaften keineswegs ein neues Phänomen, da diese Handlungen von der Gesellschaft nicht als gewerblich betrachtet wurden. Die Auflösung gewohnter Rollen kommt im Falle der P2P-Dienstleistungsplattformen erst durch den neuen Maßstab der Nutzung solcher Dienstleistungen zustande. Ein weiterer Aspekt hierbei ist die Ausweitung der Geschäftspartner von Familie, Freunden und vielleicht Freunden zweiten Grades auf jede beliebige Person, die den aktuellen Anforderungen der jeweiligen Plattform genügt.

Eine Auflösung bestehender Verhältnisse geht selten ohne Widerstände vonstatten, denn sie birgt Risiken. Traditionell gut im Markt positionierte Marktteilnehmer oder gar Marktführer spüren den Druck der neuen durch den gesellschaftlichen Wandel hervorgebrachten Konkurrenz und Geschäftsmodelle (beispielsweise im Taxigeschäft im Gegensatz zu Uber). Wenn das neue Geschäftsmodell Bedürfnisse systemisch besser $\mathrm{zu}$ befriedigen weiß und damit eine bessere Dienstleistung zu erbringen vermag, bleibt dieser Druck naturgemäß bestehen. Die traditionellen Branchenführer müssen sich entscheiden, ob sie sich dem Wandel unterwerfen oder ob sie finanzielles, politisches und soziales Wissenskapital dafür einsetzen, den laufenden gesellschaftlichen Wandel zu stoppen oder zu verzögern und so die Führungsrolle wenigstens vorübergehend zu behalten.

Die Chancen neuer Institutionen für eine Gemeinschaft oder Gesellschaft bestehen aus den daraus neu entstehenden Rollen, welche die bestehenden Rollendefinitionen zwar infrage stellen und dadurch wie beschrieben ein Risiko für Etablierte darstellen, sich aber im Lösen einiger aktueller Probleme besser bewähren und dadurch ihren Nutzern Vorteile bringen. So widersprechen Geschäftsmodelle wie sie von ebay, Uber oder Airbnb betrieben werden zwar mit dem institutionalisierten Verständnis der Trennung von Gewerbe und Privatpersonen, befriedigen aber Bedürfnisse der Konsumenten besser als die etablierten Dienstleister und stellen deren Anspruch auf die Befriedigung ebendieser Bedürfnisse dadurch infrage. Dadurch entstehen Chancen auf bessere Lösungen bekannter Probleme, unter der Voraussetzung, die institutionellen Anpassungen können in der Folge gemeistert werden.

Durch P2P-Dienstleistungsplattformen kann das institutionalisierte, soziale Rollenverständnis neu diskutiert werden, da zumindest für einen Teil der Gesellschaft offensichtlich wird, dass ein Beibehalten des Bekannten neue, attraktive Lösungen verunmöglichen würde. Die gesellschaftliche Herausforderung besteht folgerichtig darin, soziale Institutionen an die neuen Verhältnisse anzupassen, wobei die Stabilität der neu ausgerichteten Institutionen stark von einem breiten sozialen Konsens abhängt, der dem 
individuellen Anspruch auf verbesserte Problemlösung wie auch dem gesellschaftlichen Anspruch auf stabile Verhältnisse gerecht wird (Seyfert 2011).

P2P-Dienstleistungsplattformen stellen naturgemäß vor allem Institutionen im Bereich der Erwerbsarbeit infrage, allerdings nicht nur. Die nachfolgenden Beispiele sollen einen Eindruck davon vermitteln, welche Fragen institutioneller Wandel nach sich ziehen kann, auch wenn er wie in diesem Fall nur ein sehr beschränktes Gebiet betrifft.

Die Unterscheidung zwischen Angestellten und Selbstständigen ist nach herkömmlichen Maßstäben unschwer zu leisten, abgesehen vielleicht von der Kategorie der Scheinselbstständigen, die sich allerdings auch nur als Folge der weitläufigen Institutionalisierung von „Outsourcing“ etabliert hat. Doch in welche Kategorie ein Taxifahrer fällt, der zwar seinen Lohn von einer Vermittlungsplattform überwiesen bekommt, die für sich Provision einstreicht, allerdings nicht von dieser für den Job als Taxifahrer angestellt wurde, muss u. a. auch juristisch erst geklärt werden. Ob die Schaffung einer solchen neuen Kategorie Auswirkungen auf die bereits bestehenden Erwerbskategorien hat, ist dabei nicht vorwegzunehmen und zeigt den dynamischen Charakter gesellschaftlichen Wandels. Ähnliche Fragen stellen sich zu Themen wie diversen Versicherungen, Vorsorge, Reichweite der eigenen Haftpflicht, Versteuerung der Einkünfte/Umsätze, Kündigungsschutz, Verzollung von Handelsware, Nutzung von Business-to-BusinessAngeboten usw.

\subsection{3 Ökologische Aspekte}

Klimawandel und die Ausschöpfung der natürlichen Ressourcen unseres Planeten setzen Nationen vor große ökologische Herausforderungen. Der ökologische Handlungsdruck wächst und wirkt sich gleichermaßen auf Staat, Wirtschaft und Bevölkerung aus. Die Schonung der natürlichen Ressourcen steht auf der weltweiten politischen Agenda und wurde in 2015 von der Generalversammlung der UNO verabschiedete Resolution A/RES/70/1 - „Transforming our world: the 2030 Agenda for Sustainable Development" adressiert (UN DESA United Nations Department of Economic und Social Affairs 2016). In der Schweiz beschäftigt sich die Impulsgruppe Dialog Grüne Wirtschaft mit der Thematik und hält fest, dass die Menschheit daran ist, die Belastbarkeit des Planeten $\mathrm{zu}$ überschreiten und damit die Umweltbedingungen $\mathrm{zu}$ verlassen, welche für ein langfristiges Überleben der Menschheit günstig sind. Als Treiber für die Übernutzung des Planeten nennt sie das starke Anwachsen der Weltbevölkerung, die Steigerung der Wirtschaftskraft und damit des Konsumniveaus als wesentliche Einflussfaktoren auf diese Entwicklung. Das Konsumniveau in der Schweiz bildet den Großteil der Umweltbelastung in der Schweiz. Würden alle Länder pro Kopf so viel konsumieren wie die Schweiz, würde die Belastbarkeitsgrenze des Planeten massiv überschritten. Die umweltrelevantesten Konsumbereiche sind die Ernährung, das Wohnen und die Mobilität (Impulsgruppe Dialog Grüne Wirtschaft 2016). 
Die ökologischen Herausforderungen, vor denen die Menschheit steht, wurden in der Schweiz u. a. durch den Aktionsplan Grüne Wirtschaft 2013 aufgenommen. In seinem Zwischenbericht an den Bundesrat hat das Bundesamt für Umwelt (BAFU) im April 2016 Maßnahmen für eine ressourcenschonende, zukunftsfähige Schweiz formuliert. Die Maßnahmen zielen darauf $a b$, die Leistungsfähigkeit der Wirtschaft und damit die Wohlfahrt insgesamt zu stärken, indem es eine Wirtschafts- und Konsumweise fördert, welche die Knappheit begrenzter Ressourcen und die Regenerationsfähigkeit erneuerbarer Ressourcen berücksichtigt und die Ressourceneffizienz steigert. Für die Legislaturperiode 2016-2019 sieht der Bericht u. a. Sensibilisierungsmaßnahmen vor, welche ressourcenschonende Kauf- und Nutzungsentscheide fördert (Umwelt 2016). Die Impulsgruppe Dialog Grüne Wirtschaft sieht in der Sharing Economy ein verstecktes Potenzial zur Ressourcenschonung. Sie will neue Formen des Konsums fördern, indem sie Konsumenten dafür sensibilisiert und die Kreislaufwirtschaft in die Praxis umsetzt. Sie treibt damit die Idee des Nutzens statt Besitzens voran, wie sie im Rahmen der Sharing Economy bereits praktiziert wird.

P2P-Plattformen eröffnen Raum für neue Geschäftsmodelle, die das Teilen einer Ressource in den Vordergrund stellen. Geschäftsmodelle, welche ungenutzte Waren oder Kapazitäten, wie z. B. die Vermietung von ansonsten leerstehenden Räumen oder das Vermieten einer ansonsten ungenutzten Mitfahrgelegenheit, fördern die Ressourceneffizienz und können zur Veränderung in Richtung einer Kreislaufwirtschaft beitragen.

\subsection{4 Ökonomische Aspekte}

Die Entfaltung neuer Verhaltensweisen in Bezug auf Konsum und neue Beschäftigungsmöglichkeiten werden durch P2P-Plattformen geschaffen. Diese Entwicklung bietet große Chancen für eine größere Inklusion und wirtschaftliches Wachstum, stellt aber die herkömmliche Wirtschaft und auch soziale Institutionen vor neuen Herausforderungen.

\subsubsection{Veränderung im Konsumverhalten}

Der klassische Konsument war lange Zeit primär ein Verbraucher. In den westlichen, kapitalistisch geprägten Gesellschaften bemaß sich daher der gesellschaftliche Status stark an den finanziellen Möglichkeiten der Mitglieder, die sich wiederum für alle wahrnehmbar am klarsten im Ressourcenverbrauch manifestierten.

Der stärker werdende Community-Gedanke ermöglichte in den letzten Jahrzehnten allerdings alternative Statuszuschreibungen. Dies hat zur Folge, dass der Konsum der jüngeren Generationen bereits in großen Teilen anders gesehen wird, als dass dies bei der Elterngeneration noch der Fall war (Bucic et al. 2012).

Wie sich diese neuen Formen von Marktwirtschaft von den traditionellen - auf die Gewinner/Verlierer-Dichotomie fixierten - Formen unterscheiden, ist dabei abschließend nicht beurteilbar. Grund dafür ist die zunehmende Abwesenheit für große Gruppen 
gültiger Sozialskripts, die das Aufkeimen kleinerer Communities überhaupt ermöglicht hat. Diese ist aber auch dafür verantwortlich, dass sich Communities kaum mehr an einem einzigen Vorbild orientieren, sondern, gestärkt durch den Siegeszug des Individualismus eine Community nach ihren eigenen Bedürfnissen ausrichten. Dass diese Bedürfnisse sich - im Vergleich zur Elterngeneration - weniger am materiellen Erfolg (und Verbrauch) des Einzelnen orientieren, sondern das gesellschaftlich effiziente Nutzen von Ressourcen in den Mittelpunkt stellen (Ghosh 2006), mag vielerlei Motivationen haben. Zwei in diesem Zusammenhang zentrale Strömungen sind die Umweltbewegung und die Debatte um Corporate Social Responsibility (CSR). Beide bestanden zwar schon seit dem ausgehenden 19. Jahrhundert, gewannen aber erst anfangs der 1970er-Jahre an gesellschaftlicher Bedeutung (Marens 2010; Marens 2013; McCormick 1991). Obwohl ab 1987 theoretisch durch die Nachhaltigkeitsdebatte vereint, verbleibt der Umweltgedanke dominierend, was auch an der Ausrichtung der neuen Geschäftsmodelle abzulesen ist. Wenn Ressourcen geschont werden sollten, so handelt es sich zumeist um stoffliche Ressourcen und seltener um soziale Ressourcen. Das Spielfeld ist groß und damit sind die Möglichkeiten zur erfolgreichen Realisierung alternativer, kollaborativer Geschäftsmodelle sicherlich noch längst nicht ausgeschöpft.

Eine weitere Entwicklung zeichnet die Auflösung der funktionalen Differenzierung der gesellschaftlichen Sphären „Konsum“ und „Produktion“ auf. Wurde damals der Konsument als passiver Käufer und Verbraucher gesehen, nimmt er heute eine immer aktivere Rolle bei der Leistungserbringung ein. Dem sogenannten arbeitenden Konsumenten wird seit Ende der 1990er-Jahre vermehrt eine Mitverantwortung bei der Leistungserbringung übertragen, wie z. B. im E-Commerce oder E-Banking (Kleemann et al. 2009). P2P-Plattformen stützen sich teilweise stark auf den arbeitenden Konsumenten ab: Wikipedia beispielsweise, indem die Nutzenden die inhaltlichen Beiträge verfassen oder Dienstleistungsplattformen, wie z. B. Airbnb, indem Konsumenten dazu aufgerufen werden, die Leistungsbezieher $\mathrm{zu}$ bewerten. Eine Besonderheit von P2P-Plattformen in dieser Hinsicht liegt auch darin, dass der Konsument auf derselben Plattform gleichzeitig auch Produzent sein kann. So kann dieselbe Person auf derselben Plattform einmal eine Fahrgelegenheit anbieten und ein anderes Mal eine in Anspruch nehmen.

\subsubsection{Veränderungen der Anbieterlandschaft}

P2P-Plattformen erleichtern den Marktzugang für neue Leistungsanbieter. Die Eintrittshürde selbst als Leistungsanbieter auf dem Markt aufzutreten, ist dank der Existenz von geeigneten Plattformen sehr klein. Will man freien Wohnraum für Kurzaufenthalte anbieten, seine Fahrdienste für kurze Personentransporte oder seine handwerklichen Fähigkeiten für kleine Reparaturarbeiten in der Nachbarschaft zur Verfügung stellen, so genügen ein paar Klicks auf der entsprechenden Plattform, um sich als Leistungserbringer zu registrieren. Einmal registriert, wird die angebotene Leistung auf dem Markt sofort ersichtlich und für Leistungsbezieher rasch auffindbar. 
Der Anreiz, ungenutzte Kapazitäten zu monetarisieren steigt und steht für große Kreise der Gesellschaft offen. Entsprechende Leistungen auf dem Markt anzubieten, ist nunmehr nicht einem bestimmten Berufskreis vorbehalten, sondern steht auch Privatpersonen offen. Die Folge davon ist das Aufkommen einer großen Anzahl von selbstständig Erwerbstätigen auf dem Markt. Mithilfe von P2P-Plattformen können neue Einkommensquellen erschlossen werden und neue Beschäftigungschancen mit flexiblen Arbeitszeitregelungen entstehen (Europäische Kommission 2016). Der Kreis der Leistungserbringer wird damit wesentlich erweitert und auch die Vielfältigkeit der Angebote.

Wie auch der Eintritt zum Markt, gestaltet sich die Änderung oder auch der Rückzug eines Angebots vom Markt dank P2P-Plattformen sehr einfach. Dank dieser Einfachheit können Leistungserbringer ihr Angebot flexibel an ihre eigenen Kapazitäten oder an die Nachfrage anpassen. Sind die Kapazitäten ausgeschöpft, kann der Leistungserbringer leicht sein Angebot reduzieren oder zurückziehen. Sieht der Leistungsanbieter seine Teilnahme auf der P2P-Plattform als eine Möglichkeit, sich einen Nebenverdienst zu verschaffen (opportunity providers), kann er im Falle einer Verschlechterung der Bedingungen auf der Plattform mit einem Austritt reagieren. Zunehmend sind auf P2P-Plattformen jedoch Leistungsanbieter unterwegs, für die die Einnahmen aus der Plattform die primäre Einkommensquelle darstellen (necessity providers). Ihre Teilnahme an P2P-Plattformen ist weniger von den Bedingungen auf der Plattform selbst als von der Gesamtwirtschaftslage abhängig. Eröffnen sich dem Leistungserbringer Beschäftigungsmöglichkeiten außerhalb der Plattformwirtschaft, wird er diese nutzen (EPRS European Parliamentary Research Service 2016).

Der einfache Marktzutritt und -austritt wirkt sich auf den bestehenden Markt aus und kann die Angebotslandschaft verändern, indem die bestehenden Leistungen durch mehr oder neuartige Leistungen erweitert wird, bestehende Leistungen wegfallen oder das bestehende Leistungsangebot durch neue Leistungsangebote substituiert wird. Diese Veränderungen durch P2P-Plattformen beeinflusst auch die Nachfrageseite. Die Erweiterung des Angebots ist für die Leistungsempfänger von Vorteil, da die Angebote oft ihre Bedürfnisse besser abdecken und preisgünstiger sind. Für Leistungserbringer stellen sich dadurch jedoch Fragen rund um die Sozialleistungen. Für bereits im Markt etablierte Akteure stellen diese neuen Leistungserbringer eine neue Herausforderung dar, welche die bestehenden Verhältnisse teilweise grundlegend verändern.

\subsubsection{Kommerzialisierung bisheriger Praktiken des Teilens}

Teilen ist ein Phänomen, das es in unserem gesellschaftlichen Umgang immer schon gegeben hat. Ein prominentes Beispiel ist das Teilen von Landmaschinen. Bauern investieren gemeinsam in Landmaschinen, die sie nur einige Male im Jahr nutzen, und teilen sich die Maschinen dann. Dies wird schon lange so praktiziert und erfolgt auf persönlicher Kommunikationsebene, da die Bauern i. d. R. benachbart sind und teilweise auch schon über Generationen von der Option des Teilens Gebrauch machen. Auch die 
schon lange existierenden Wohngemeinschaften sind ein Beispiel dafür, dass das Phänomen des Teilens kein neuer Gedanke ist.

Neu ist allerdings, dass durch die Möglichkeiten, die die digitale Transformation bietet, sich solche einfachen Praktiken des Teilens mittlerweile in deutlich größeren Dimensionen und vor allem über größere Distanzen realisieren lassen. Hieraus entstand in den letzten zwei Jahrzehnten ein neues Geschäftsmodell in Form von Online-Plattformen, was den ursprünglichen Tauschgedanken erweitert und eine Kommerzialisierung des Tauschs von Dienstleistungen und Gütern herbeiführte. Standen beim ursprünglichen Gedanken des Teilens neben einer kosteneffizienten Lösung der Aspekt der Nachhaltigkeit und der soziale Aspekt häufig im Vordergrund, ist heutzutage ganz klar zu beobachten, dass der Trend stark geworden ist, aus kommerzieller Motivation heraus zu teilen (Hamari et al. 2016).

Auch Airbnb, eines der prominentesten Beispiele für eine erfolgreiche P2P-Plattform zur Wohnungsvermittlung, entstand, indem zwei Mitgründer von Airbnb in finanzieller Not waren und ein freistehendes Zimmer in ihrer Wohnung an Reisende vermittelten. Da schnell klar war, dass die Nachfrage und Zahlungsbereitschaft bei einem solchen Konzept sehr groß ist, war das neue Geschäftsmodell- über eine P2P-Plattform freistehende Zimmer und Wohnungen zu vermitteln - schnell geboren (Hofmann 2016). Die Alliance for a New Economy (LAANE) zeigt auf, dass der Großteil des Umsatzes der Plattform heute durch kommerzielle Anbieter generiert wird (Samaan 2015).

In Deutschland sind bereits über 100 Online-Plattformen verfügbar, auf denen P2PSharing-Dienstleistungen angeboten werden. Dabei liegt der Fokus mittlerweile auf Mobilität und Gebrauchsgegenständen (vgl. Abb. 3.1). Einen großen Teil machen dabei Angebote zu Co-Using und Verkauf aus.

Abb. 3.2 stellt die Neuregistrierungen von P2P-Plattformen dar, die in Deutschland genutzt werden können. Hier ist klar zu erkennen, dass ein besonderer Hype für neue P2P-Plattformen in den Jahren 2010-2014 existierte und danach die Neuanmeldungen drastisch zurückgingen. Erste Plattformen in den 1990er-Jahren waren ebay und Homeexchange.
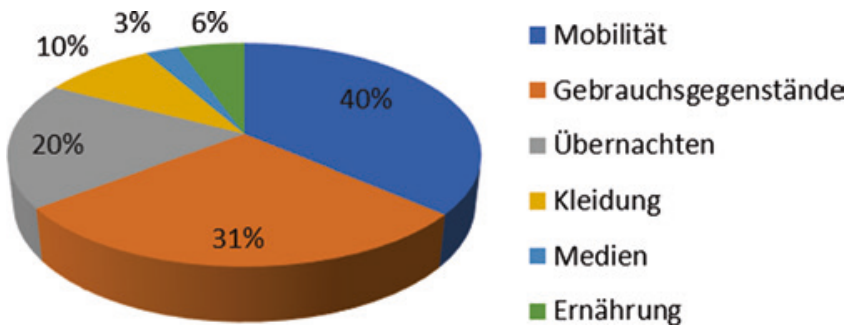

Abb. 3.1 Anzahl von P2P-Plattformen in Deutschland nach Konsumbereichen. (Eigene Darstellung; nach: Harnisch 2017) 


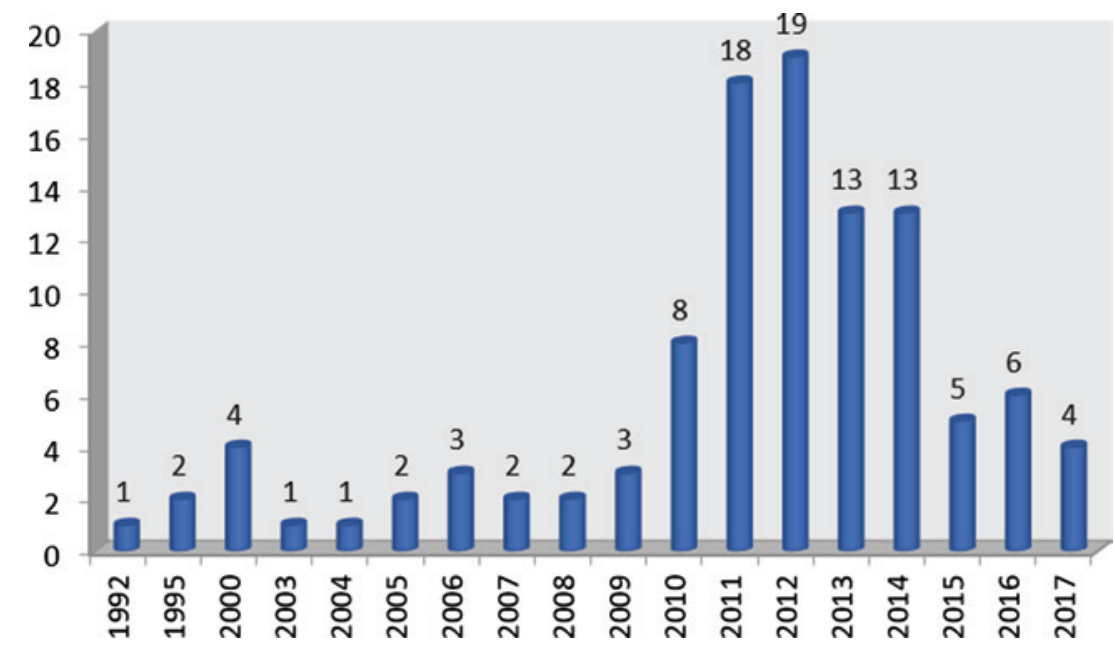

Abb. 3.2 Anzahl von Neugründungen von P2P-Plattformen in Deutschland. (Eigene Darstellung; nach: Harnisch 2017)

\subsection{Chancen und Herausforderungen für die Gesellschaft durch P2P-Dienstleistungsplattformen}

P2P-Dienstleistungsplattformen bergen für eine Gesellschaft neben zahlreichen Chancen auch einige Herausforderungen. Im Folgenden werden einige ausgewählte Chancen und Herausforderungen betrachtet, die bei P2P-Dienstleistungen von zentraler Bedeutung sind.

\subsubsection{Chancen durch P2P-Plattformen}

\section{Zeit- und ortsunabhängige Dienstleistungsangebote}

Durch die Digitalisierung besteht die Chance, möglichst viele Personen unabhängig von Ort und Zeit zu erreichen und somit bei P2P-Dienstleistungen aus der Perspektive von Leistungsbezügern ein reichhaltiges Angebot von Dienstleistungen zu erhalten. Aus der Sicht von Leistungserbringern kann über P2P-Plattformen eine einfache und schnelle Kommunikation zu potenziellen Kunden aufgebaut werden.

\section{Erweiterung der Angebotslandschaft}

Durch die direkte Vermittlung zwischen Peers können Leistungen, die üblicherweise über Vermittlungsfirmen oder andere Institutionen (traditionelle Intermediäre) angeboten wurden, innovativer gestaltet werden. Prominentes Beispiel hierfür sind P2P-Kredite. 
Erfolgte die Vergabe von Krediten traditionell über Kreditinstitute, können Kreditgeber und Kreditnehmer mittels P2P-Plattformen, wie z. B. zopa.com auf Finanzinstitute als Akteur verzichten. Dadurch eröffnen sich neue Möglichkeiten der Finanzierung, wie z. B. crowd-lending. Auch im Verkehrsbereich setzen sich neue innovative Modelle zunehmend durch. Waren für die Personenbeförderung im Fernverkehr üblicherweise Busgesellschaften oder öffentliche Verkehrsmittel die herkömmliche Anlaufstelle, können heute alternativ dazu auch P2P-Plattformen, welche Mitfahrgelegenheiten vermitteln, genutzt werden.

\section{Volkswirtschaftliches Wachstum}

Das Ausmaß des volkswirtschaftlichen Potenzials, das durch P2P-Plattformen geschaffen wird, ist schwierig einzuschätzen. Jedoch wird ihr ein enormes Potenzial für volkswirtschaftliches Wachstum zugeschrieben. Gemäß Studien der Europäischen Kommission konnte in 2013 dank P2P-Plattformen weltweit ein Zusatzeinkommen von 5,5 Mrd. US\$ generiert werden. Der Umsatz aus P2P-Plattformen für 2013 wird weltweit auf 20 Mrd. US\$ geschätzt (EPRS European Parliamentary Research Service 2016).

Die im vorangehenden Kapitel beschriebenen Veränderungen legen ein weiteres Wachstum von auf P2P-Plattformen basierende Geschäftsmodelle nahe. Eine Einschätzung des European Parliamentary Research Services (EPRS) ergibt, dass fast die Hälfte der für den Privatkonsum hergestellten Waren und Dienstleistungen potenziell über P2P-Plattformen angeboten werden könnten (EPRS European Parliamentary Research Service 2016). P2P-Plattformen würden so den Konsum bestimmter Waren und Dienstleistungen ankurbeln, auch von solchen, die auf den herkömmlichen Märkten fehlen.

\section{Professionalisierung bisheriger sozialer Praktiken}

Eine Professionalisierung sozialer Praktiken bringt zumeist eine Standardisierung gewissen Grades mit sich, um die Dienstleistung replizierbar und damit planbar zu halten. Dies wiederum erleichtert es anderen, die gleiche oder zumindest eine ähnliche Dienstleistung anzubieten, was die Erreichbarkeit der Dienstleistung stark erhöht.

Zum Beispiel: Durch den Großteil der Menschheitsgeschichte war Altenpflege eine soziale Praxis, die nahezu ausschließlich innerhalb der Kerngruppe angefragt und geleistet wurde. Hatte jemand aus irgendwelchen Gründen keinen Anschluss an solch eine Kerngruppe, war Altenpflege kaum erreichbar. Erst durch die moderne Nachfrage nach Altenpflege in der Nachkriegszeit des 20. Jahrhunderts als Folge demografischer und medizinischer Entwicklungen wurde diese Dienstleistung professionalisiert und steht seitdem einem immer breiterem Publikum offen, sodass Altenpflege heutzutage nicht mehr gezwungenermaßen vom Sozialnetz abhängt und somit auch alleinstehende ältere Menschen Aussicht auf einen umsorgten Lebensabend haben. 


\subsubsection{Herausforderungen durch P2P-Plattformen}

\section{Vertrauensverlust durch Anonymität in einer virtuellen Welt}

In herkömmlichen geschäftlichen Beziehungen sind die Akteure den persönlichen und physischen Kontakt gewöhnt und können sie so auf zwischenmenschlicher Ebene eine Vertrauensbasis schaffen. Durch die digitale Transformation und den zunehmenden Trend, geschäftliche Beziehungen auf Online-Plattformen abzuwickeln, entfällt der persönlichen Kontakt und damit die Vertrauensgrundlage. Sowohl für Leistungserbringer als auch für Leistungsempfänger müssen neue Dienste geschaffen werden, die ein solches Vertrauen auch auf Online-Plattformen herstellen. Vernetzungen über soziale Netzwerke, Bewertungssysteme und das Einbinden von vertrauenswürdigen Stellen sind Beispiele, dem Phänomen der Anonymität entgegenzuwirken. So bietet beispielsweise Airbnb die Möglichkeit an, dass Fotos des offerierten Objekts von zertifizierten Stellen gemacht werden, um so das Vertrauen in die Echtheit der Fotos zu stärken.

Neben der Gefahr von Vertrauenseinbußen in den jeweiligen Geschäftspartner durch die im digitalen Business vorhandene Anonymität besteht die Unsicherheit für Nutzende von P2P-Dienstleistungsplattformen bezüglich der Verarbeitung ihrer Daten auf solchen Plattformen. Digitale Plattformen speichern die Daten ihrer Nutzer in der Regel und verwenden sie u. a. für zielgerichtete Werbung (targeted advertising). Für die Nutzenden von Dienstleistungsplattformen und damit auch von P2P-Plattformen ist es nach wie vor kaum nachvollziehbar, was mit ihren Daten geschieht. Auch dieses Phänomen birgt einen Vertrauensverlust für Nutzende von P2P-Plattformen in das digitale Geschäftsmodell gegenüber traditionellen Geschäftsmodellen mit sich.

\section{Schwächung der gesellschaftlichen Strukturen}

Eine Professionalisierung sozialer Praxen kann zu einer leichteren Erreichbarkeit der Dienstleistung führen, da sie nicht mehr vom Grad des Sozialanschlusses abhängt. Dasselbe Phänomen kann allerdings auch schnell dazu führen, dass eine Praxis, z. B. die Altenpflege, nicht mehr als Teil der institutionalisierten Rolle der Familie gesehen, sondern als normale Dienstleistung empfunden wird. Hat jemand die finanzielle Mittel nicht, sich eine solche Dienstleistung zu erwerben, kann er nicht mehr auf den automatischen Rückhalt seiner Kerngruppe zählen, selbst wenn diese vorhanden wäre. Die Professionalisierung der Altenpflege hat diese aus dem Selbstverständnis der Kerngruppe entfernt, was zu einer Schwächung der sozialen Strukturen innerhalb von Kerngruppen führt. Pointiert formuliert sind die Mitglieder einer Gruppe weniger aufeinander angewiesen, wenn Dienstleistungen von außen bezogen werden können und sehen sich daher auch weniger in der gegenseitigen Verantwortung.

\section{Neue Formen des Arbeitens}

Verschiedene Formen des Arbeitens waren lange Zeit stabil institutionalisiert. Die neue Unsicherheit, ob Arbeitsformen der privaten oder der gewerblichen Sphäre zuzuordnen sind, birgt Herausforderungen für alle Beteiligten. Ohne eine klare Unterscheidung, 
fehlt dem Erbringer der Dienstleistung die Gewissheit, welche Steuern und Abgaben zu entrichten sind und welche Freiheiten bzw. Pflichten ihm zustehen. Abgesehen von der lähmenden Wirkung von Unsicherheiten auf die Wirtschaft, tendieren unregulierte Situationen dazu, das Recht des Stärkeren zu begünstigen. Im hochregulierten Verhältnis von Arbeitnehmer und Arbeitgeber ist ein Rückzug darauf allerdings unakzeptabel, weshalb staatliche Institutionen bemüht sind, diese zeitnah klar unterscheiden zu können. Orientierungspunkte für die Differenzierung gibt die Europäische Kommission in den überarbeiteten Leitlinien für die Richtlinie über unlautere Geschäftspraktiken. Dabei empfiehlt sie die Prüfung der Häufigkeit der Dienstleistungen, die Gewinnorientierung und die Umsatzhöhe. Eine bestimmte Kombination kann darauf hinweisen, dass es sich bei einem Anbieter um einen Gewerbetreibenden handelt. Klare Regelungen und Kategorien fehlen allerdings derzeit.

\section{Konkurrenz zu herkömmlichen Branchen}

Bieten die Leistungsanbieter auf P2P-Plattformen Leistungen an, die anderweitig auch angeboten werden, so stellen sie eine direkte Konkurrenz zu diesen Anbietern dar. Dies ist z. B. der Fall in der Übernachtungsbranche oder in der Taxibranche: P2P-Plattformen, welche Fahrgelegenheiten anbieten, substituieren die Angebote in der Taxibranche; P2P-Plattformen, welche Übernachtungsmöglichkeiten für Kurzaufenthalte anbieten, substituieren die Angebote auf dem Hotelbranche. Anbieter, welche ihre Leistungen nicht mittels P2P-Plattformen anbieten, werfen der Regierung ungleiche Spieße vor. So seien die Leistungsanbieter auf P2P-Plattformen nicht an dieselben Auflagen gebunden wie sie und erfahren deshalb einen Vorteil.

P2P-Plattformen können sich auch auf die Branche auswirken, indem sie Angebote, die anderweitig nachgefragt werden, für sich beanspruchen. So hat Samaan (2015) festgestellt, dass die auf Airbnb angebotenen Liegenschaften zu einer Abnahme der für die städtische Bevölkerung zur Verfügung stehenden Liegenschaften geführt hat.

Dass sich P2P-Plattformen auf die herstellende Industrie der auf der Plattform angebotenen Güter auswirken, ist naheliegend. Fördern P2P-Plattformen doch die Wiederverwendung von einmal gekauften Gütern, ist die daraus resultierende Folge eine Abnahme der Nachfrage nach diesen Gütern. Studien belegen eine Reduktion der Anzahl Fahrzeugbesitzer in Zusammenhang mit Car-Sharing-Angeboten (Cervero et al. 2007; Martin et al. 2010).

\section{Rebound-Effekte}

Im Rahmen plattformbasierter Dienstleistungen wird sehr häufig ein positiver Einfluss hinsichtlich Ressourcenschonung vorangestellt. Insbesondere bei Dienstleistungsmodellen wie Car-Sharing wird dies sehr gerne als ein wesentliches Argument für die Stärkung solcher Konzepte angeführt. Werden Autos geteilt, so impliziert dies zunächst, dass weniger Autos genutzt werden, was eine positive Auswirkung auf die Umwelt haben sollte. Bedenkt man jedoch, dass eine Ausbreitung des Car-Sharing-Konzepts möglichweise dazu führt, dass immer mehr Personen die Dienstleistungen von 
Car-Sharing-Anbietern beziehen und damit aber auch weniger öffentliche Verkehrsmittel nutzen, stellt sich die Frage, ob die positiven Einflüsse bezüglich Ressourcenverbrauch wirklich vorhanden sind. Dieser Effekt wird als Rebound-Effekt bezeichnet. Es handelt sich dabei um einen Effekt, bei dem das vermeintliche Einsparpotenzial von Effizienzsteigerung nur bedingt existiert, da es durch andere Faktoren nahezu aufgehoben wird (Thomas 2012).

\subsection{Ausblick}

Dieser Beitrag zeigt auf, dass der gesellschaftliche Boden für P2P-Plattformen, der von vorangegangen Tendenzen zur Individualisierung und Wertfreiheit geschaffen wurde, gesellschaftliche Entwicklungen in diese Richtung weiter vorantreibt und damit einen breiten Einfluss auf die Wirtschaft aufweist. Während P2P-Plattformen das Gefühl einer Gemeinschaftszugehörigkeit zu stärken vermögen, kann auch festgestellt werden, dass Gruppen, welche die Zugangshürden, z. B. finanzieller, technischer oder sprachlicher Art, nicht meistern, von dieser Gemeinschaft eher ausgeschlossen werden. Die zunehmende Nutzung von P2P-Plattformen eröffnet die Möglichkeit, die Potenziale der P2P-Dienstleistungsplattformen auszuschöpfen, wobei die mit der Nutzung dieser Plattformen verbundenen Unsicherheiten sich aktuell noch als große Hürde herausstellen. Die neuen Beschäftigungschancen, die sich dank P2P-Plattformen ergeben, stehen Rechtsunsicherheiten in Bezug auf die soziale Sicherheit gegenüber. Die Idee der Förderung der schonenderen Nutzung von Ressourcen kann durch Rebound-Effekte kontraproduktive Wirkungen zeitigen.

Staatsregierungen sehen in einer sogenannten P2P-Economy ein großes Potenzial für wirtschaftliches Wachstum und eine effizientere Nutzung von natürlichen Ressourcen. Sowohl in der Schweiz wie auch auf EU-Ebene sollen Maßnahmen ergriffen werden, die es erlauben dieses Potenzial voll auszuschöpfen.

\section{Literatur}

BAFU Bundesamt für Umwelt. (2016). Bericht an den Bundesrat. Grüne Wirtschaft. Massnahmen des Bundes für eine ressourcenschonende, zukunftsfähige Schweiz. https://www.uvek.admin.ch/ uvek/de/home/umwelt/gruene-wirtschaft.html. Zugegriffen: 5. Apr. 2019.

Beck, U. (1986). Risikogesellschaft: Auf dem Weg in eine andere Moderne. Frankfurt a. M.: Suhrkamp.

Belk, R. (2014). You are what you can access: Sharing and collaborative consumption online. Journal of Business Research, 67(8), 1595-1600.

BITKOM. (2013). Das Internet schafft eine Kultur des Teilens. https://www.bitkom.org/Presse/ Presseinformation/Das-Internet-schafft-eine-Kultur-des-Teilens.html. Zugegriffen: 16. Aug. 2016. 
BMWi Bundesministerium für Wirtschaft und Energie. (2016). Grünbuch Digitale Plattformen. http://www.bmwi.de/BMWi/Redaktion/PDF/G/gruenbuch-digitale-plattformen,property $=$ pdf,bereich $=$ bmwi2012, sprache $=$ de,rwb=true.pdf. Zugegriffen: 18. Dez. 2016.

Bodendorf, F. (1999). Wirtschaftsinformatik im Dienstleistungsbereich. Berlin: Springer.

Botsman, R., \& Rogers, R. (2010). What's mine is yours - How collaborative consumption is changing the way we live. New York: HarperBusiness.

Bucic, T., Harris, J., \& Arli, D. (2012). Ethical consumers among the millennials: A cross-national study. Journal of Business Ethics, 110(1), 113-131.

Cervero, R., Golub, A., \& Nee, B. (2007). City carshare: Longer-term travel demand and car ownership impacts. Transportation Research Record: Journal of the Transportation Research Board, 1992(1), 70-80.

Chtacherbia, E., Mittermeier, L., Oberhausen, R., \& Wieland, T. (2002). Peer to Peer in Theorie und Praxis. JavaSpectrum, 04(2002), 23-28.

Dahrendorf, R. (1961). Gesellschaft und Freiheit - Zur soziologischen Analyse der Gegenwart. München: Piper.

Deaux, K. (1996). Social identification. In E. T. Higgins \& A. W. Kruglanksi (Hrsg.), Social psychology: Handbook of basic principles (S. 777-798). New York: Guilford.

EPRS European Parliamentary Research Service. (2016). The cost of Non-Europe in the sharing economy. Economic, social and legal challenges and opportunities. http://www.europarl.europa. eu/RegData/etudes/STUD/2016/558777/EPRS_STU(2016)558777_EN.pdf. Zugegriffen: 10. Jan. 2017.

Europäische Kommission. (2016). Europäische Agenda für die kollaborative Wirtschaft. https:// ec.europa.eu/transparency/regdoc/rep/1/2016/DE/1-2016-356-DE-F1-1.PDF. Zugegriffen: 2. Juni 2016.

Eurostat. (2015). Households with internet access at home. http://ec.europa.eu/eurostat/web/ products-datasets/-/tin00088. Zugegriffen: 20. Juli 2016.

Fourastié, J. (1954). Die große Hoffnung des 20. Jahrhunderts. Köln-Deutz: Bund-Verlag.

Gottmann, J. (1957). Megalopolis or the urbanization of the Northeastern seaboard. Economic Geography, 33(3), 189-200.

Hamari, J., Sjöklint, M., \& Ukkonen, A. (2016). The sharing economy: Why people participate in collaborative consumption. Journal of the Association for Information Science and Technology, 7(9), 2047-2059.

Harnisch, R. (2017). Landkarte des online-gestützten Peer-to-Peer Sharing in Deutschland 2017. http://www.peer-sharing.de/veroeffentlichungen/landkarte-peer-to-peer-sharing.html. Zugegriffen: 5. Apr. 2019.

Hill, B. (2010). The sociology of innovation. Cambridge. MIT. http://mako.cc/academic/generals/ bmh-generals-sociology_innovation.pdf. Zugegriffen: 4. Aug. 2016.

Hillmann, K. (1994). Wörterbuch der Soziologie. Stuttgart: Kroener.

Hofmann, A. (2016). Der Airbnb-Gründer über die Geschichte hinter seinem Milliarden-Startup. http://www.gruenderszene.de/allgemein/nathan-blecharczyk-airbnb-interview/2. Zugegriffen: 25. Apr. 2016.

Impulsgruppe Dialog Grüne Wirtschaft. (2016). Go for impact. https://www.gruenewirtschaft. admin.ch/grwi/de/home/go-for-impact/Go-for-Impact.html. Zugegriffen: 10. Jan. 2017.

ITU. (2017). ICT Facts and figures 2017. https://www.itu.int/en/ITU-D/Statistics/Documents/facts/ ICTFactsFigures2017.pdf. Zugegriffen: 10. Jan. 2017.

Kardorff, E. (2006). Virtuelle Netzwerke - Eine Neue Form Der Vergesellschaftung? In B. Hollstein \& F. Straus (Hrsg.), Qualitative Netzwerkanalyse (S. 63-97). Wiesbaden: VS Verlag.

Keller, A. (2013). Teilen heisst das neue Haben. Absatzwirschaft, 56(6), 32-37. 
Kleemann, F., Voß, G., \& Rieder, K. (2009). Crowdsourcing und der Arbeitende Konsument. Sozialwissenschaftlicher Fachinformationsdienst. Industrie- und Betriebssoziologie, 2009(1), 9-23.

Marens, R. (2010). Destroying the village to save it: Corporate social responsibility, labour relations, and the rise and fall of American hegemony. Organization, 17(6), 743-766.

Marens, R. (2013). What comes around: The early 20th century American roots of legitimating corporate social responsibility. Organization, 20(3), 454-476.

Martin, S., Shaheen, A., \& Lidicker, J. (2010). Impact of carsharing on household vehicle holdings. Transportation Research Record: Journal of the Transportation Research Board, 2143(1), 150-158.

McCormick, J. (1991). Reclaiming paradise: The global environmental movement. Bloomington: Indiana University Press.

Mead, G. (1934). Mind, self, and society. Chicago: University of Chicago Press.

OECD. (2014). Measuring the digital economy: A new perspective. http://www.oecd-ilibrary.org/ science-and-technology/measuring-the-digital-economy_9789264221796-en. Zugegriffen: 10. Jan. 2017.

Popper, K. (1975). Die offene Gesellschaft und ihre Feinde. München: Francke.

Quinn, J. B. (1992). Intelligent enterprise: A knowledge and service based paradigm. United States of America: Free Press.

Redlich, T., \& Wulfsberg, J. (2011). Wertschöpfung in der Bottom-up-Ökonomie. Heidelberg: Springer.

Samaan, R. (2015). IAANE policy brief: Short-term rentals and Los Angeles' lost housing. http:// www.laane.org/wp-content/uploads/2015/08/Short-Term_RentalsLAs-Lost_Housing.pdf. Zugegriffen: 2. Jan. 2017.

Seyfert, R. (2011). Das Leben der Institutionen: Zu einer allgemeinen Theorie der Institutionalisierung. Weilerswist: Velbrück.

Stalder, F. (2016). Kultur der Digitalität. Frankfurt a. M.: Surkamp.

The Tomás Rivera Policy Institute. (2015). Access \& use of technology - Disparities in access and use of information technology. http://immigrantservices.uscmediacurator.com/disparities-in-access-and-use-of-information-technology-it/. Zugegriffen 29. Dez. 2016.

Thomas, S. (2012). Energieeffizienz spart wirklich Energie - Erkenntnisse zum Thema „ReboundEffekte“. Energiewirtschaftliche Tagesfragen, 62(8), 8-11.

Thome, H. (1985). Wandel zu postmaterialistischen Werten?: Theoretische und empirische Einwände gegen Ingleharts Theorie-Versuch. Soziale Welt, 36(1), 27-59.

Tönnies, F. (1887). Gemeinschaft und Gesellschaft: Abhandlung des Communismus und des Socialismus als empirische Culturformen. Leipzig: Fues's.

Traiblmaier, H. (2006). Datenqualität und individualisierte Kommunikation. Wiesbaden: Deutscher Universitätsverlag.

UN DESA United Nations Department of Economic and Social Affairs. (2016). Sustainable development goals. https://sustainabledevelopment.un.org/sdgs\#. Zugegriffen: 10. Jan. 2017.

Warschauer, M. (2004). Technology and social inclusion: Rethinking the digital divide. Cambridge: Mass. \& MIT.

Weber, M. (1922). Wirtschaft und Gesellschaft. Tübingen: Mohr.

Weller, I. (2015). Freizeit und Lebensqualität in der Postkonsumgesellschaft. In R. Freericks \& D. Brinkmann (Hrsg.), Handbuch Freizeitsoziologie (S. 255-275). Wiesbaden: Springer. 
Weissenfeld, Katinka (Dipl. Wirtsch. Ing./katinka.weissenfeld@bfh.ch) Dozentin am Institut Public Sector Transformation des Departements Wirtschaft der Berner Fachhochschule für Projektmanagement und Wirtschaftsinformatik. Mehrjährige Projektmanagementaufgaben im IT-Umfeld. Forschungsschwerpunkte E-Accessibility, Privacy, Web Technologien.

Dungga, Angelina (lic.phil.hist./angelina.dungga@bfh.ch) Research Associate am Institut für Public Sector Transformation des Departements Wirtschaft der Berner Fachhochschule. Forschungsinteresse: Public Sector Innovation, E-Inclusion. Dozentin für empirische Sozialforschung und digitale Transformation in Gesellschaft und Politik. Davor Leiterin Dienst Nomenklaturen im Bundesamt für Statistik der Schweiz.

Frecè, Jan Thomas (Dr. des./jan.frece@bfh.ch) Lehr- und Forschungstätigkeit in den Themengebieten dezentrale Identitäten und Daten, digitale Transformation, nachhaltige Unternehmensführung und Unternehmenswerte in den Instituten Public Service Transformation und Sustainable Business der Berner Fachhochschule, davor Projekte zur betrieblichen Nachhaltigkeit am Institut für Unternehmensführung der Fachhochschule Nordwestschweiz, davor Project Manager, Service Solution Architect und Root Cause Analyst bei IBM Schweiz.

Open Access Dieses Kapitel wird unter der Creative Commons Namensnennung 4.0 International Lizenz (http://creativecommons.org/licenses/by/4.0/deed.de) veröffentlicht, welche die Nutzung, Vervielfältigung, Bearbeitung, Verbreitung und Wiedergabe in jeglichem Medium und Format erlaubt, sofern Sie den/die ursprünglichen Autor(en) und die Quelle ordnungsgemäß nennen, einen Link zur Creative Commons Lizenz beifügen und angeben, ob Änderungen vorgenommen wurden.

Die in diesem Kapitel enthaltenen Bilder und sonstiges Drittmaterial unterliegen ebenfalls der genannten Creative Commons Lizenz, sofern sich aus der Abbildungslegende nichts anderes ergibt. Sofern das betreffende Material nicht unter der genannten Creative Commons Lizenz steht und die betreffende Handlung nicht nach gesetzlichen Vorschriften erlaubt ist, ist für die oben aufgeführten Weiterverwendungen des Materials die Einwilligung des jeweiligen Rechteinhabers einzuholen.

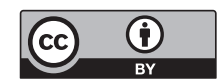

